\title{
XSEDE Integration with ORCID for Research Resources
}

\author{
David L. Hart \\ National Center for Atmospheric \\ Research \\ Boulder, CO USA \\ dhart@ucar.edu
}

Steven Peckins

National Center for

Supercomputing Applications

speckins@illinois.edu

\author{
Ester Soriano \\ National Center for Supercomputing \\ Applications \\ University of Illinois at Urbana- \\ Champaign \\ Urbana, IL USA \\ esoriano@illinois.edu \\ Rob Light \\ Pittsburgh Supercomputing Center \\ Pittsburgh, PA USA \\ light@psc.edu
}

\author{
Carrie Arnold \\ Texas Advanced Computing Center \\ University of Texas at Austin \\ Austin, TX USA \\ cmarnold@tacc.utexas.edu
}

\author{
Burt Cubbison \\ Pittsburgh Supercomputing Center \\ cubbison@psc.edu
}

\author{
Josh Berger \\ National Center for Atmospheric Research \\ jberger@ucar.edu
}

\author{
Michael Shapiro \\ National Center for Supercomputing Applications \\ shapiro2@illinois.edu
}

\begin{abstract}
Many research infrastructure providers face the challenge of identifying the science output of their user communities. The XSEDE Resource Allocations Service (XRAS) has recently completed integration with the ORCID ecosystem, allowing XSEDE to post allocation award information to the ORCID profiles of its users. Through these ORCID-posted awards, publishers will be able to link users' papers to the XSEDE-allocated projects that supported the research. To support the integration, XSEDE staff enhanced the user portal and XRAS and performed some novel administrative steps. Other XRAS client organizations can take advantage of these features to connect their allocation awards to their users' ORCID profiles.
\end{abstract}

\section{CCS CONCEPTS}

- Information systems $\sim$ Reputation systems $・$ Information systems $\sim$ Web services • Social and professional topics $\sim$ Professional topics

\section{KEYWORDS}

XSEDE, ORCID, Research resources, Allocations, Interoperability

Permission to make digital or hard copies of part or all of this work for personal or classroom use is granted without fee provided that copies are not made or distributed for profit or commercial advantage and that copies bear this notice and the full citation on the first page. Copyrights for third-party components of this work must be honored. For all other uses, contact the Owner/Author.

PEARC '19, July 28-August 1, 2019, Chicago, IL, USA

(C) 2019 Copyright is held by the owner/author(s).

ACM ISBN 978-1-4503-7227-5/19/07.

https://doi.org/10.1145/3332186.3333252

\section{Introduction}

ORCID allows any researcher to create a long-term, persistent, and unique identifier for themselves; ORCID IDs persist across job changes, name changes, and different naming styles used by publishers. Employers, publishers, and funding agencies have long been able to update the ORCID profiles of their employees, authors, and awardees, respectively. Most recently, ORCID introduced the ability to add research resource awards (e.g., XSEDE allocation awards) to researcher profiles. The XSEDE team recently implemented this ORCID feature within its portal and the XSEDE Resource Allocation Service (XRAS) [1].

XSEDE's integration with the ORCID ecosystem benefits users, XSEDE, Service Providers, publishers, and ORCID. Users benefit from a more complete range of research activities in their ORCID profiles. XSEDE and Service Providers gain greater insight into their impact on science. Publishers gain a more consistent mechanism for describing the support for an author's publications. And ORCID extends its vision for the research ecosystem, in which "all who participate in research, scholarship, and innovation are uniquely identified and connected to their contributions across disciplines, borders, and time" [2].

\section{Organizational Prerequisites}

To take advantage of the XRAS-ORCID features, your organization must have two administrative items in place. Keep in mind that two organizational entities are involved here: (a) the organization posting the information to ORCID, and (b) the organization hosting the resources. These may be the same or different organizations or sub-organizations. For XSEDE, XSEDE is posting allocation awards to ORCID, and the Service Providers are associated with their respective resources. 
First, the organization posting to ORCID profiles must be an ORCID member [3]. XSEDE worked with ORCID to adapt their member agreement for a virtual organization, since XSEDE agreements must be signed by the University of Illinois. If your institution is already an ORCID member, you're all set.

Second, your organization - both the posting organization and the resource host organization, if different-needs to have a unique identifier in either the Global Research Identifier Database (GRID) [4] or RINGGOLD systems [5]. To complete this step, XSEDE requested an identifier (as a subunit of the University of Illinois) from the GRID system at no cost (Figure 1). Most universities already have entries in both systems; however, through GRID, you may also request an identifier for a subunit of a parent organization, for example, a research computing unit within a university.

\section{Research Resources Configuration}

The organization hosting the resources can control how it wants its resource information to appear in the ORCID posts. You use XSEDE's Resource Description Repository (RDR) [6] to configure a few key settings to manage this feature.

First, you must update your service provider information to include your organization's GRID identifier. The information page for your

\section{Extreme Science and Engineering Discovery Environment} grid.501421.3

\section{Figure 1: XSEDE's unique identifier in the GRID system.}

service provider has an "External Data Posting" section where you enter this information.

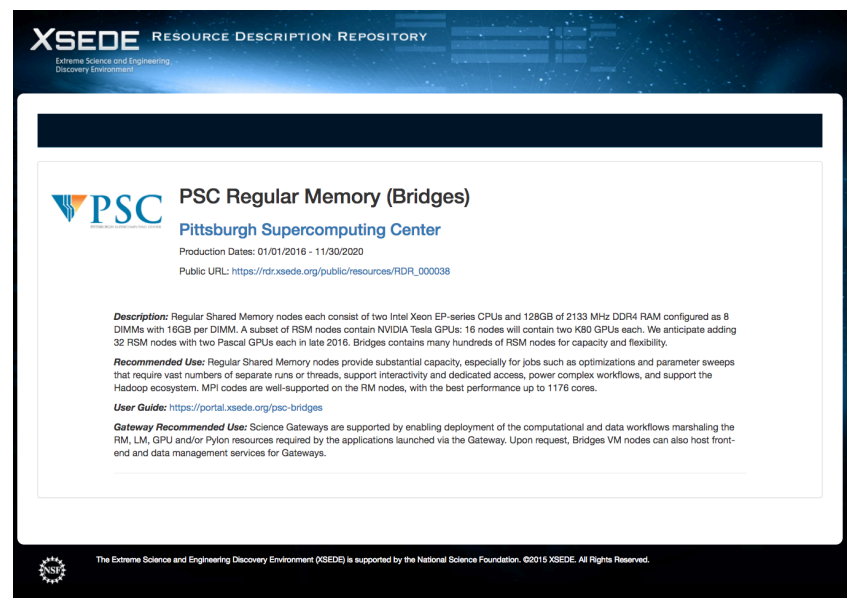

Figure 2: A resource's public page in the XSEDE Resource Description Repository.
Second, ORCID requires a unique, resolvable identifier for each resource that you want included in the posts to ORCID. For various reasons, you may want to feature certain resources and not others. In RDR, you configure each resource by visiting its edit page. In the "Resource Publishing" section, you can check a box to allow RDR to publish a public page for the resource (Figure 2). The resource will only be posted to ORCID if this box is checked. By default the public identifier and page will be generated by RDR, but if you prefer, you may enter your own resolvable URL to be used when posting the resource to ORCID. If you provide your own URL, it should refer to a persistent identifier that will not go away when the resource is decommissioned.

\section{Linking to ORCID IDs}

Users are central to the ORCID ecosystem, and you must provide a means for your users to link their ORCID identities to your organization's user identities. In the same step, users grant their permission for your organization to post information to their ORCID profiles. ORCID's model ensures that you will update profiles only for users who agree to have the information posted.

The linking step for XSEDE is handled within the XSEDE User Portal [7]. The basic process is similar to the process used when you, for example, link accounts when using Facebook or Google to authenticate to another site. After linking, the user permission granted by the user - in the form of an ORCID access token - can be passed to XRAS via the XRAS client's identity service API, through which XRAS gathers user information. Users can revoke these permissions at any time.

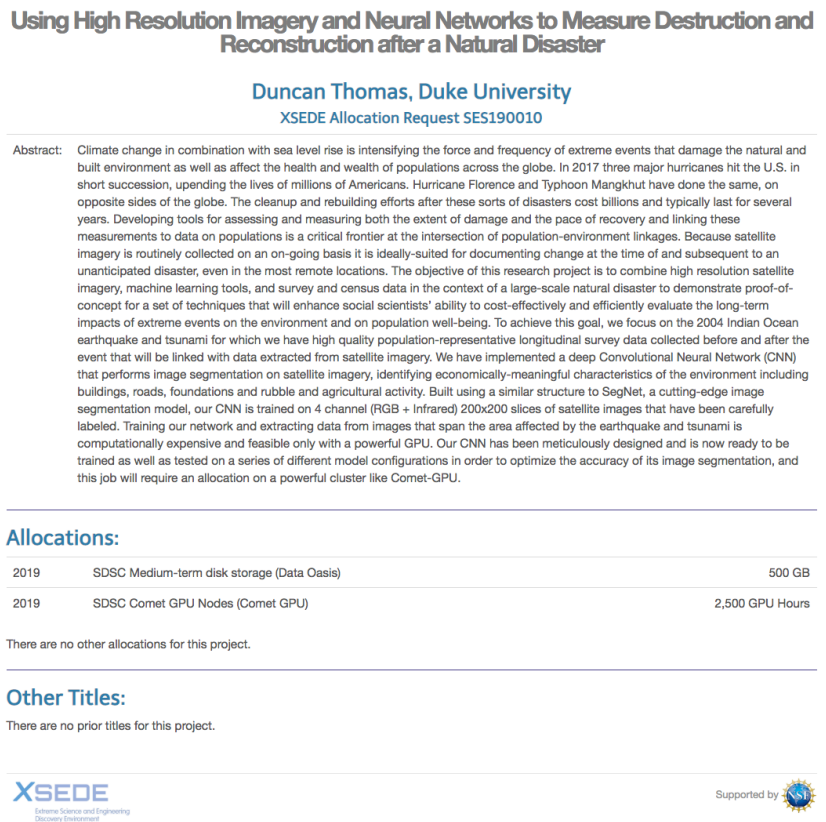

Figure 3: An example XRAS-hosted public page for an allocation award. 


\section{XRAS Configuration}

Through XRAS, you post allocation awards to ORCID on behalf your organization. As noted, ORCID requires a unique organization ID, and you enter this information via the XRAS Admin interface, under Client Settings. You must also allow XRAS to host a public page for any approved projects that are posted to ORCID. You can "Enable Public Pages" for those allocation types you want posted to ORCID (Figure 3). Once the public pages have been turned on, you can post individual awarded projects or all awarded projects for a certain opportunity.

XRAS will post to the ORCID research-resources API when an allocation has been approved and when user permissions have been granted (Figure 4). The project metadata includes the research proposal title, the allocation begin and end dates, the allocated resource(s) that have been configured for ORCID posting in RDR,

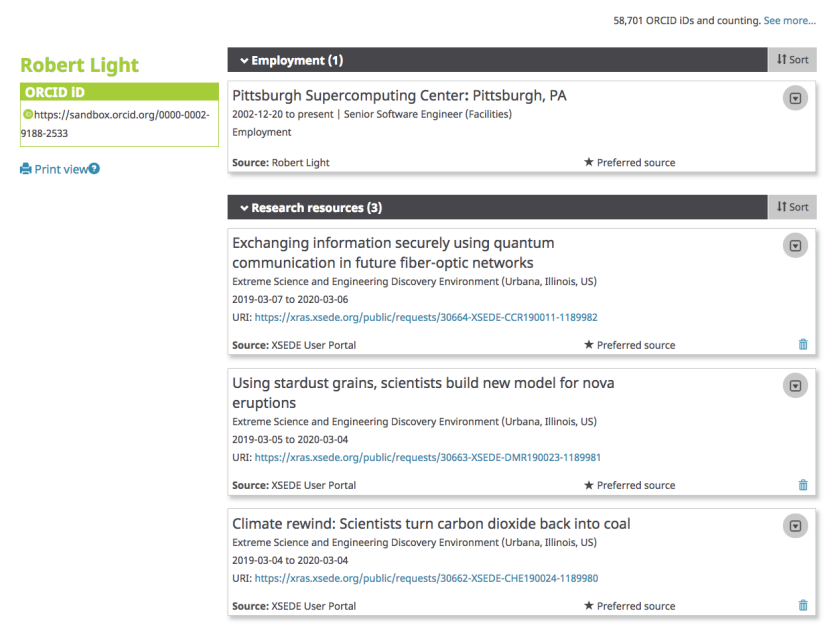

Figure 4: Example XSEDE-allocated projects posted to a user's profile in ORCID's testing site. links to the public pages for each of those resources, and a pointer to the XRAS public page for more details.

\section{Conclusions}

The XSEDE and XRAS work was completed in January 2019, and so far more than 400 users have linked their ORCID IDs to their XSEDE user profiles. XSEDE continues to promote this opportunity and plans to begin posting allocation awards to ORCID profiles in mid-2019. Complementing this work, in February 2019, version 1.2 of the Journal Publishing Tag Set [8] was released. Part of the Journal Article Tag Suite (JATS), the Publishing Tag Set is the metadata schema used by publishers to formally describe articles. Version 1.2 includes support for identifying "nonmonetary resources," effectively providing a means for publishers to link XSEDE allocation and similar resource awards that supported the research in an article.

\section{ACKNOWLEDGMENTS}

This work used the Extreme Science and Engineering Discovery Environment (XSEDE), which is supported by National Science Foundation grant number OAC-1548562. This material is based upon work supported by the National Center for Atmospheric Research, which is a major facility sponsored by the National Science Foundation under Cooperative Agreement No. 1852977.

\section{REFERENCES}

[1] XSEDE. 2018. About XRAS. Retrieved May 2019 from https://portal.xsede.org/allocations/about-xras.

[2] ORCID. What is ORCID. Retrieved May 2019 from https://orcid.org/about.

[3] ORCID. ORCID Member Organizations. Retrieved May 2019 from https://orcid.org/members.

[4] Global Research Identifier Database. 2018. GRID: Cataloging the world's research organizations. Retrieved May 2019 from https://www.grid.ac.

[5] Ringgold, Inc. 2019. RINGGOLD: Organizing the World. Retrieved May 2019 from https://www.ringgold.com/.

[6] XSEDE. 2015. XSEDE Resource Description Repository. Retrieved May 2019 from https://rdr.xsede.org/.

[7] XSEDE. 2019. XSEDE User Portal. Retrieved May 2019 from https://portal.xsede.org.

[8] National Center for Biotechnology Information. 2019. Journal Publishing Tag Set: Version 1.2. $\quad$ Retrieved May 2019 from https://jats.nlm.nih.gov/publishing/1.2/. 